\title{
Biological Evaluation and Mechanistic Studies of Quinolin-(1H)- Imines as a New Chemotype against Leishmaniasis
} \author{
Ana S. Ressurreição, ${ }^{d}$ (D) Ana M. Tomás ${ }^{a, e}$ \\ ai3S, Instituto de Investigação e Inovação em Saúde, Porto, Portugal \\ bIBMC, Instituto de Biologia Molecular e Celular, Porto, Portugal \\ ‘Centro de Engenharia Biológica, Universidade do Minho, Braga, Portugal \\ diMed.ULisboa, Research Institute for Medicines, Faculty of Pharmacy, Universidade de Lisboa, Lisbon, Portugal \\ eICBAS, School of Medicine and Biomedical Sciences, Universidade do Porto, Porto, Portugal
}

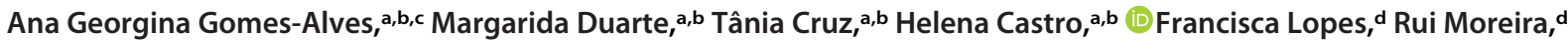

ABSTRACT Leishmaniasis is one of the most challenging neglected tropical diseases and remains a global threat to public health. Currently available therapies for leishmaniases present significant drawbacks and are rendered increasingly inefficient due to parasite resistance, making the need for more effective, safer, and less expensive drugs an urgent one. In our efforts to identify novel chemical scaffolds for the development of antileishmanial agents, we have screened in-house antiplasmodial libraries against axenic and intracellular forms of Leishmania infantum, Leishmania amazonensis, and Leishmania major. Several of the screened compounds showed half-maximal inhibitory concentrations $\left(\mathrm{IC}_{50} \mathrm{~S}\right)$ against intracellular $L$. infantum parasites in the submicromolar range (compounds $1 \mathrm{~h}, \mathrm{IC}_{50}=0.9 \mu \mathrm{M}$, and $1 \mathrm{n}, \mathrm{IC}_{50}=0.7 \mu \mathrm{M}$ ) and selectivity indexes of 11 and 9.7, respectively. Compounds also displayed activity against L. amazonensis and L. major parasites, albeit in the low micromolar range. Mechanistic studies revealed that compound $1 \mathrm{n}$ efficiently inhibits oxygen consumption and significantly decreases the mitochondrial membrane potential in L. infantum axenic amastigotes, suggesting that this chemotype acts, at least in part, by interfering with mitochondrial function. Structure-activity analysis suggests that compound $1 \mathrm{n}$ is a promising antileishmanial lead and emphasizes the potential of the quinoline- $(1 H)$-imine chemotype for the future development of new antileishmanial agents.

KEYWORDS Leishmania, antiparasitic agents, mechanisms of action, quinoline-(1H)imine chemotype

eishmaniases are a group of neglected diseases caused by trypanosomatid parasites of the genus Leishmania that manifest in several clinical forms, including cutaneous leishmaniasis, the most common form, mucocutaneous leishmaniasis, a highly disfiguring and debilitating condition, and visceral leishmaniasis, a life-threatening disorder. According to the latest reports, the World Health Organization (WHO) estimates that approximately 1 billion people, mainly from the poorest communities in tropical and subtropical countries, live at risk of developing these illnesses (1). In the absence of a human vaccine or a prophylactic drug, control of leishmaniases is largely based on chemotherapy of infected individuals. Current available medicines include first-line agents, such as pentavalent antimonials (sodium stibogluconate and meglumine antimoniate), which are administered daily over the course of 20 to 30 days, and secondline agents (amphotericin B, pentamidine, miltefosine, and paromomycin), which are often used in unresponsive or resistant cases (2). However, none of these drugs has even close to optimal performance, and their use in leishmaniasis treatment is compromised by several factors: high costs, prolonged administration over multiple weeks,

Citation Gomes-Alves AG, Duarte M, Cruz T, Castro H, Lopes F, Moreira R, Ressurreição AS, Tomás AM. 2021. Biological evaluation and mechanistic studies of quinolin-(1H)-imines as a new chemotype against leishmaniasis. Antimicrob Agents Chemother 65:e01513-20. https://doi.org/10.1128/AAC.01513-20. Copyright $\odot 2021$ American Society for Microbiology. All Rights Reserved. Address correspondence to Ana S. Ressurreição, aressurreicao@ff.ulisboa.pt, or Ana M. Tomás, atomas@ibmc.up.pt.

Received 17 July 2020 Returned for modification 22 October 2020 Accepted 6 April 2021

Accepted manuscript posted online 26 April 2021

Published 17 June 2021 
and poor safety indexes, presenting important adverse effects (2). Miltefosine, for instance, the only available oral drug for treatment of leishmaniasis, is unsuitable for pregnant patients due to fetotoxic and teratogenic risks (3). A further problem limiting the efficacy of therapy in areas of endemicity is the gradual emergence of parasite strains resistant to antileishmanial drugs (4).

The multiple drawbacks associated with current leishmaniasis therapeutics, together with the worldwide public health impact of these diseases, prompts the scientific community to identify new chemotypes for developing effective, safe, and accessible antileishmanial drugs (5). Quinolines and structurally related heterocycles are privileged scaffolds found in several natural products that exhibit a wide range of biological properties, including activity against different Leishmania spp. (in both in vitro and in vivo models), as well as against other protozoa (6). The antiplasmodial 8-aminoquinolines sitamaquine (SQ) and tafenoquine (TFQ), for example, have been extensively studied as potential treatments for visceral leishmaniasis. From a mechanistic point of view, TFQ appears to suppress the parasite's respiratory chain through cytochrome $c$ reductase (complex III) inhibition, inducing a rapid drop in intracellular ATP and depolarization of mitochondrial membrane potential (7). SQ, on the other hand, was reported to inhibit succinate dehydrogenase (complex II), an essential enzyme for Leishmania (8). Quinolin-4(1H)-imines, which can be considered tautomeric analogues of quinolines, were previously reported by some of us as potent dual-stage antiplasmodials, displaying potential to bind to the $\mathrm{Q}_{0}$ ubiquinone oxidation site of cytochrome $b c_{1}$ from Plasmodium falciparum $(9,10)$. More recently, quinolin- $4(1 H)$ imines were also disclosed as efficient antitrypanosomal agents, endowed with excellent pharmacokinetic profiles (11). Additionally, a high-throughput phenotypic screen of roughly 600,000 small molecules against Leishmania mexicana promastigotes identified a quinolin-4(1H)-imine derivative as one of the top hits (12), further highlighting the potential of this chemotype against parasitic diseases. In this paper, we report the screening of a set of quinolin-4(1H)-imines from in-house antiplasmodial libraries against promastigotes and amastigotes of three different Leishmania species. Our results show that several compounds inhibit parasitic growth within mammalian host macrophages and that the mechanism of action can be explained, to some extent, by their ability to interfere with Leishmania parasites' mitochondrial function.

\section{RESULTS}

Effect of chemical libraries on $L$. infantum proliferation: structure-activity relationships (SARs). The leishmanicidal potential of 38 compounds was initially assessed at a single concentration against $L$. infantum promastigotes (10 or $20 \mu \mathrm{M}$ ) and axenic amastigotes $(10 \mu \mathrm{M})$. With the purpose of increasing the chemical diversity of our initial screening, besides the main family of quinolin-4(1H)-imines (compounds $1 a$ to $-n$ and $2 a$ to $-m$ ), we also evaluated the structurally related pyridon- $4(1 H)$-imines (compounds $3 a$ to $-c$ ), as well as flavones (compounds $4 a$ to $-\mathrm{h}$ ), bioisosteres of the quinolonimine core scaffold $(13,14)$.

Inspection of the activity data presented in Table 1 reveals that pyridon-4(1H)imines and flavones are not effective against $L$. infantum promastigotes and are poorly active against axenic amastigotes (inhibition of $<30 \%$ at $10 \mu \mathrm{M}$ ). In contrast, the vast majority of quinolin-4(1H)-imines tested were found to be active against the promastigote stage of the parasite (inhibition of $\geq 90 \%$ at 10 or $20 \mu \mathrm{M}$ ), with several compounds also exhibiting good activity against axenic amastigotes (inhibition of $\geq 30 \%$ at $10 \mu \mathrm{M}$ ). Among the quinolin-4(1H)-imine family, preliminary structure-activity relationships indicate that the size and nature of the $N$-linked side chain is important for activity in both stages of the parasite. With the exception of compound $2 \mathrm{c}$, quinolin- $4(1 \mathrm{H})$-imines belonging to class 1 ( $\mathrm{X}=$ methyl or ethyl) are more potent against axenic amastigotes than compounds containing a basic alkylamino side chain at the $\mathrm{N}-1$ nitrogen atom of the quinolonimine scaffold (class 2 ). Therefore, quinolin- $4(1 H)$-imines 1 e to $-\mathrm{n}$, presenting good inhibition profiles (promastigotes, $\geq 90 \%$, and axenic amastigotes, $\geq 30 \%$ ) were selected for $\mathrm{IC}_{50}$ determination for the same parasite forms, as well as for intramacrophagic amastigotes. Quinolin-4(1H)-imines $1 \mathrm{~d}$ and $2 \mathrm{c}$ were also included for comparative purposes. The cytotoxicities of the different molecules to BMDM were 
TABLE 1 Anti-Leishmania activities of chemical libraries against $L$. infantum promastigotes and axenic amastigotes ${ }^{a}$

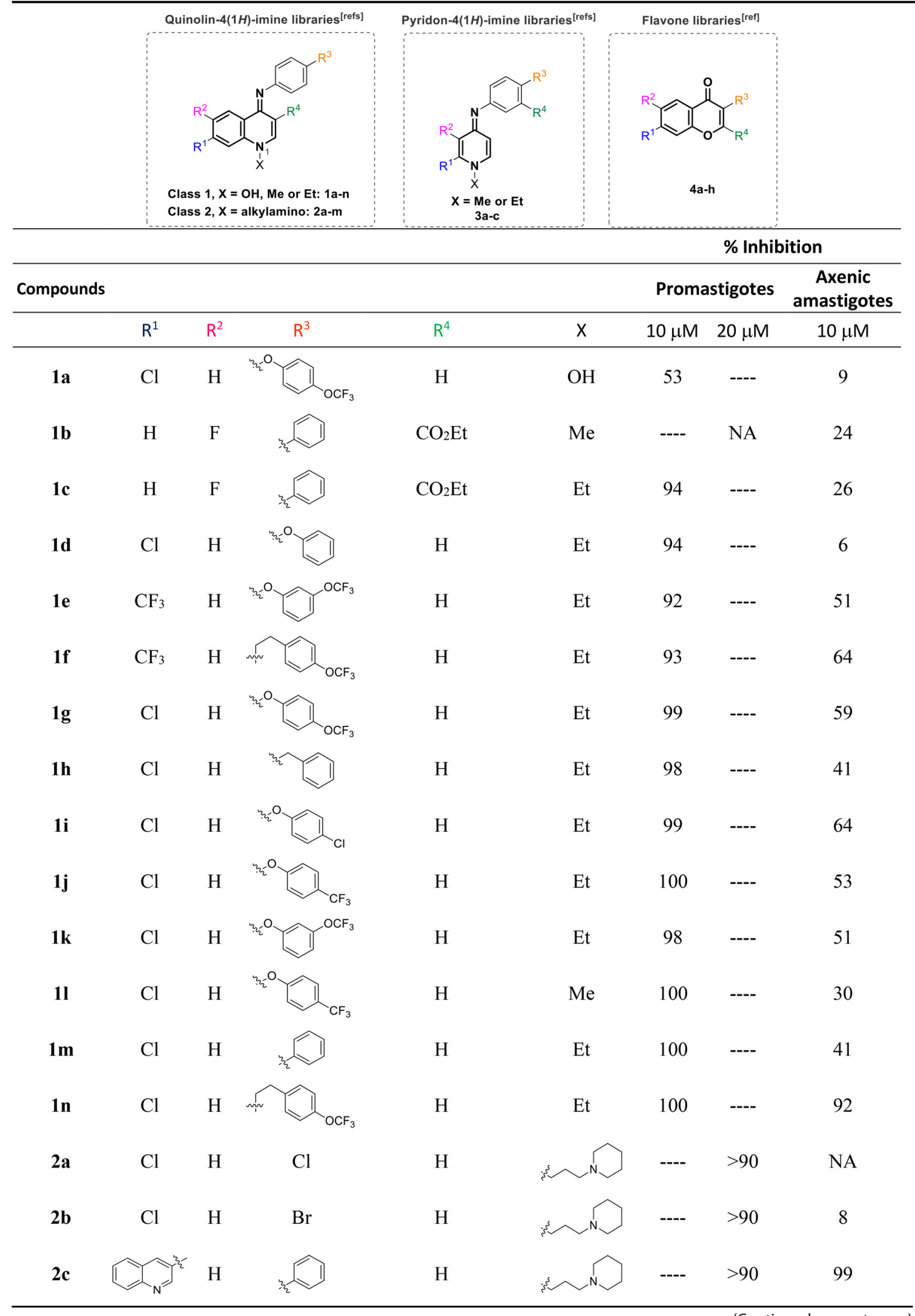




\begin{tabular}{|c|c|c|c|c|c|c|c|c|}
\hline $2 d$ & $\mathrm{Cl}$ & $\mathrm{H}$ & $\mathrm{Me}$ & $\mathrm{H}$ & & ---- & $>90$ & 12 \\
\hline $2 e$ & $\mathrm{Cl}$ & $\mathrm{H}$ & & $\mathrm{H}$ & & 90 & ---- & 20 \\
\hline $2 f$ & $\mathrm{Cl}$ & $\mathrm{H}$ & & $\mathrm{H}$ & & 100 & ---- & NA \\
\hline $2 g$ & $\mathrm{CF}_{3}$ & $\mathrm{H}$ & & $\mathrm{H}$ & & 100 & ---- & NA \\
\hline $2 h$ & $\mathrm{CF}_{3}$ & $\mathrm{H}$ & & $\mathrm{H}$ & & 100 & ---- & 23 \\
\hline $2 i$ & $\mathrm{Cl}$ & $\mathrm{H}$ & & $\mathrm{H}$ & & 100 & ---- & 21 \\
\hline $2 j$ & $\mathrm{H}$ & $\mathrm{CF}_{3}$ & & $\mathrm{H}$ & & 100 & ---- & 33 \\
\hline $2 k$ & $\mathrm{CF}_{3}$ & $\mathrm{H}$ & & $\mathrm{H}$ & & 100 & ---- & 26 \\
\hline 21 & $\mathrm{Cl}$ & $\mathrm{H}$ & & $\mathrm{H}$ & & 100 & ---- & NA \\
\hline $2 m$ & $\mathrm{Cl}$ & $\mathrm{H}$ & & $\mathrm{H}$ & & 100 & ---- & 25 \\
\hline $3 a$ & $\mathrm{H}$ & $\mathrm{H}$ & $\mathrm{OMe}$ & $\mathrm{H}$ & $\mathrm{Me}$ & NA & ---- & 15 \\
\hline $3 b$ & $\mathrm{H}$ & $\mathrm{H}$ & $\mathrm{OH}$ & & $\mathrm{Me}$ & NA & ---- & 5 \\
\hline $3 c$ & $\mathrm{H}$ & $\mathrm{NH}_{2}$ & & $\mathrm{H}$ & --- & 45 & ---- & 79 \\
\hline $4 a$ & $\mathrm{H}$ & $\mathrm{H}$ & $\mathrm{H}$ & & --- & ---- & NA & 22 \\
\hline $4 b$ & $\mathrm{H}$ & $\mathrm{H}$ & $\mathrm{H}$ & & ---- & NA & --- & 32 \\
\hline $4 c$ & $\mathrm{H}$ & $\mathrm{H}$ & I & $\mathrm{H}$ & ---- & NA & ---- & 29 \\
\hline $4 d$ & $\mathrm{Me}$ & $\mathrm{Cl}$ & $\mathrm{H}$ & & ---- & 22 & ---- & 20 \\
\hline $4 e$ & $\mathrm{Me}$ & $\mathrm{Cl}$ & $\mathrm{H}$ & & ---- & NA & ---- & 24 \\
\hline $4 f$ & $\mathrm{CH}_{2} \mathrm{Br}$ & $\mathrm{Cl}$ & $\mathrm{Br}$ & & ---- & NA & ---- & 9 \\
\hline $4 g$ & $\mathrm{H}$ & $\mathrm{H}$ & & $\mathrm{H}$ & ---- & NA & ---- & 12 \\
\hline $4 h$ & $\mathrm{Cl}$ & $\mathrm{H}$ & $\mathrm{H}$ & & ---- & NA & ---- & 15 \\
\hline
\end{tabular}

aparasites were incubated for $24 \mathrm{~h}$ with different compounds. NA, not active. 
TABLE 2 Leishmanicidal activities for different forms of $L$. infantum and cytotoxicities of quinolin-4(1H)-imine derivatives

\begin{tabular}{|c|c|c|c|c|c|}
\hline \multirow[b]{3}{*}{ Compound } & \multicolumn{4}{|c|}{ Mean value \pm SD for ${ }^{a}$ : } & \multirow[b]{3}{*}{$\mathrm{SI}^{b}$} \\
\hline & \multicolumn{3}{|c|}{$\mathrm{IC}_{50}(\mu \mathrm{M})$ against: } & \multirow[b]{2}{*}{$\begin{array}{l}\mathrm{CC}_{50}(\mu \mathrm{M}) \text { in } \\
\mathrm{BMDM}\end{array}$} & \\
\hline & Promastigotes & Axenic amastigotes & $\begin{array}{l}\text { Intramacrophagic } \\
\text { amastigotes }\end{array}$ & & \\
\hline$\overline{1 d}$ & $2.8 \pm 0.6$ & $19.4 \pm 1.4$ & $1.3 \pm 0.5$ & $10.1 \pm 3.4$ & 7.8 \\
\hline $1 e$ & ND & $11.3 \pm 1.1$ & $2.2 \pm 0.6$ & $14.4 \pm 1.9$ & 6.5 \\
\hline $1 f$ & $4.5 \pm 0.3$ & $12.2 \pm 1.9$ & $1.7 \pm 0.4$ & $10.8 \pm 0.9$ & 6.4 \\
\hline $1 \mathrm{~g}$ & $2.3 \pm 0.2$ & $11.4 \pm 0.8$ & $1.4 \pm 0.6$ & $9.6 \pm 0.9$ & 6.9 \\
\hline $1 \mathrm{~h}$ & $1.5 \pm 0.3$ & $10.9 \pm 0.5$ & $0.9 \pm 0.1$ & $10.0 \pm 1.8$ & 11.1 \\
\hline $1 \mathrm{i}$ & $1.4 \pm 0.1$ & $5.8 \pm 2.8$ & $1.1 \pm 0.3$ & $9.1 \pm 1.4$ & 8.3 \\
\hline $1 \mathrm{j}$ & $1.9 \pm 0.1$ & $14.1 \pm 4.2$ & $1.4 \pm 0.3$ & $9.5 \pm 1.0$ & 6.8 \\
\hline $1 \mathrm{k}$ & $2.50 \pm 0.01$ & $11.0 \pm 1.7$ & $1.9 \pm 0.5$ & $10.9 \pm 1.5$ & 5.7 \\
\hline 11 & $2.42 \pm 0.02$ & $15.8 \pm 2.3$ & $1.9 \pm 0.2$ & $10.8 \pm 1.0$ & 5.7 \\
\hline $1 \mathrm{~m}$ & $1.7 \pm 0.2$ & $13.4 \pm 3.0$ & $1.3 \pm 0.2$ & $8.4 \pm 2.1$ & 6.5 \\
\hline $1 n$ & $1.9 \pm 0.2$ & $6.5 \pm 2.8$ & $0.7 \pm 0.2$ & $6.8 \pm 0.8$ & 9.7 \\
\hline $2 c$ & ND & ND & $>0.5$ & $<0.5$ & \\
\hline $\mathrm{AMB}^{\mathrm{C}}$ & $0.17 \pm 0.09$ & $0.39 \pm 0.24$ & $0.06 \pm 0.01$ & $3.1 \pm 0.6$ & 52 \\
\hline
\end{tabular}

a L. infantum promastigotes, axenic amastigotes, and intramacrophagic amastigotes, as well as BALB/C mouse BMDM, were incubated for $24 \mathrm{~h}$ with different concentrations of the different compounds. $I C_{50}$, half-maximal inhibitory concentration; $\mathrm{CC}_{50}, 50 \%$ cytotoxic concentration; ND, not determined.

${ }^{b} \mathrm{SI}$, selectivity index (ratio between $\mathrm{CC}_{50}$ and $\mathrm{IC}_{50}$ values for intramacrophagic amastigotes).

${ }^{\mathrm{C}} \mathrm{AMB}$, amphotericin $\mathrm{B}$, used as a positive control.

evaluated in parallel (Table 2). We found that the $\mathrm{IC}_{50} \mathrm{~s}$ were dependent on the parasite stage. Axenic amastigotes were the less susceptible forms $\left(\mathrm{IC}_{50} \mathrm{~s}\right.$ varying between 5.9 and $19.4 \mu \mathrm{M})$, promastigotes ranked second ( $\mathrm{IC}_{50}$ s ranging from 1.4 to $\left.4.5 \mu \mathrm{M}\right)$, and intracellular amastigotes were the most susceptible forms $\left(\mathrm{IC}_{50} \mathrm{~s}\right.$ between 0.7 and $2.2 \mu \mathrm{M})$. The 12 quinolin- $4(1 \mathrm{H})$-imines analyzed exhibited selectivity indexes (SIs) ranging between 5.7 and 11.1 (Table 2).

In a second step of the analysis, we tested compounds $1 \mathrm{~d}, 1 \mathrm{~g}, 1 \mathrm{~h}$, and $1 \mathrm{n}$ against L. major and L. amazonensis, two species causing cutaneous leishmaniasis. As shown in Table 3, intracellular amastigotes of $L$. major and $L$. amazonensis showed $I C_{50} s$ between 2.3 and $3.9 \mu \mathrm{M}$, indicating that, although these species are less sensitive to the tested quinolin-4(1H)-imines than $L$. infantum, the chemotype has therapeutic potential for both types of leishmaniases. Curiously, when the screening was carried out on promastigotes, a different result was found, as in this case, L. major was more susceptible than the other two species.

Effect of quinolin-4(1H)-imines on mitochondrial functions. Quinolin-4(1H)imines were originally designed to inhibit Plasmodium mitochondrial respiration, more particularly the cytochrome $b c_{1}$ complex, also known as complex III. To investigate whether the mitochondrial activity of Leishmania was altered by these compounds, we analyzed the effects of the compounds with better selectivity indexes on basal oxygen

TABLE 3 Effects of selected quinolin-4(1H)-imines against promastigotes and intramacrophagic amastigotes of $L$. major and $L$. amazonensis

\begin{tabular}{|c|c|c|c|c|}
\hline \multirow[b]{3}{*}{ Compound } & \multicolumn{4}{|c|}{ Mean $\mathrm{IC}_{50} \pm \mathrm{SD}(\mu \mathrm{M})(\mathrm{SI})$ against $^{a}:$} \\
\hline & \multicolumn{2}{|c|}{ Promastigotes } & \multicolumn{2}{|c|}{ Intramacrophagic amastigotes } \\
\hline & L. major & L. amazonensis & L. major & L. amazonensis \\
\hline $1 \mathrm{~d}$ & $0.5 \pm 0.1$ & $8.7 \pm 1.6$ & $3.9 \pm 1.1(2.6)$ & $2.8 \pm 1.1(3.6)$ \\
\hline $1 \mathrm{~g}$ & $0.8 \pm 0.1$ & $3.4 \pm 0.8$ & $3.4 \pm 0.9(2.8)$ & $3.5 \pm 1.3(2.7)$ \\
\hline $1 \mathrm{~h}$ & $0.6 \pm 0.2$ & $4.9 \pm 1.4$ & $2.6 \pm 0.9(3.8)$ & $2.7 \pm 0.9(3.7)$ \\
\hline $1 \mathrm{n}$ & $0.75 \pm 0.01$ & $2.4 \pm 0.6$ & $2.3 \pm 1.1(2.9)$ & $2.3 \pm 0.4(2.9)$ \\
\hline
\end{tabular}

a L. major and L. amazonensis promastigotes and intramacrophagic amastigotes were incubated for $24 \mathrm{~h}$ with serial dilutions of selected quinolin- $4(1 \mathrm{H})$-imines. $\mathrm{IC}_{50}$, half-maximal inhibitory concentration; $\mathrm{SI}$, selectivity index (ratio between $\mathrm{CC}_{50}$ and $\mathrm{IC}_{50}$ values). 


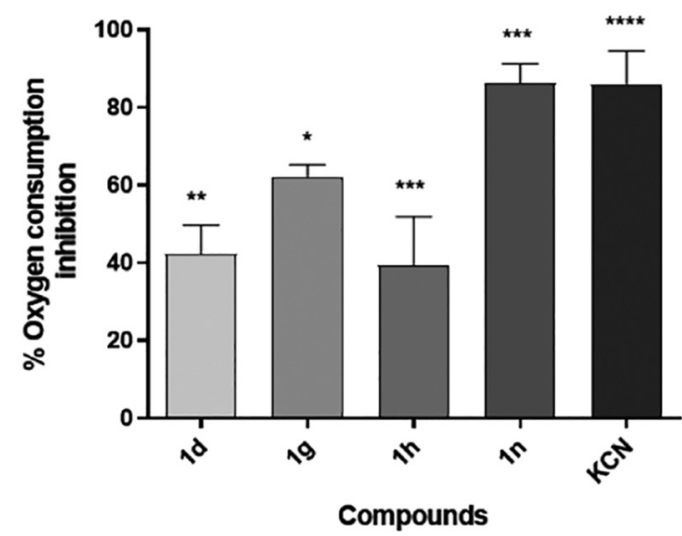

C

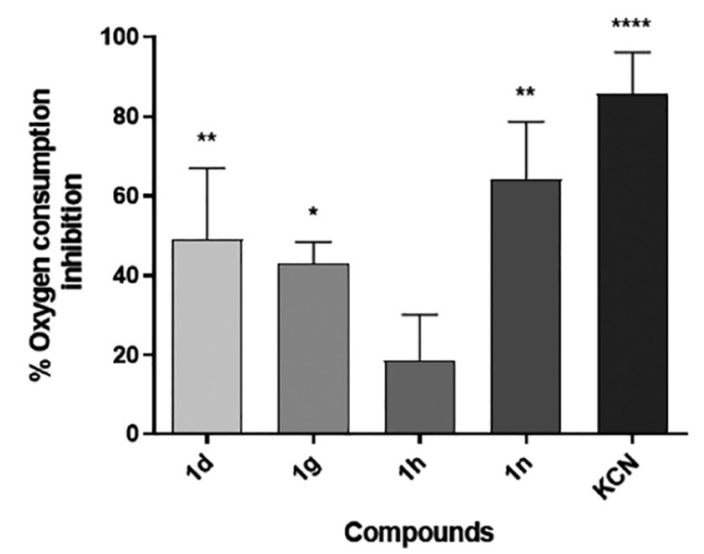

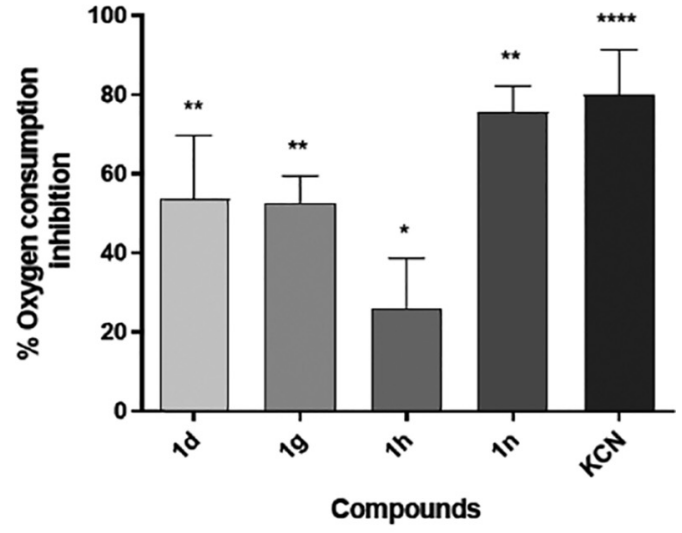

D

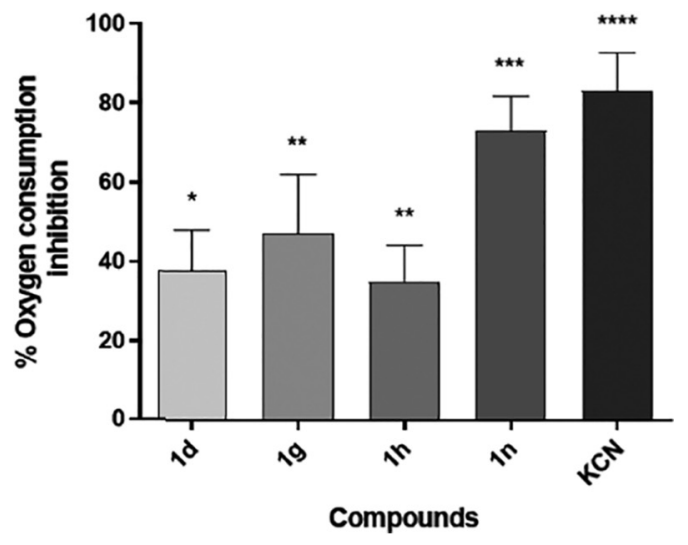

FIG 1 Effects of selected quinolin-4(1H)-imines on oxygen consumption of Leishmania parasites. L. infantum axenic amastigote (A) and L. infantum (B), L. amazonensis (C), and L. major (D) promastigotes were treated with 2 -fold the $I C_{50} S$ of each selected quinolin- $4(1 H)$-imine. Percentages of inhibition are relative to the oxygen consumption values obtained at basal levels. The graphs show the mean values and standard deviations (error bars) from 3 independent experiments. KCN was used as the positive control. Statistical significance was determined using a paired $t$ test. ${ }^{*}, P<0.05 ;{ }^{* *}, P<0.005 ;{ }^{* *}, P<0.0005 ;{ }^{* * * *}, P<0.0001$.

consumption, mitochondrial membrane potential, and reactive oxygen species (ROS) production.

Quinolin-4(1H)-imines inhibit oxygen consumption in Leishmania. The basal oxygen consumption of intact promastigotes and of axenic amastigotes was evaluated upon incubation of parasites with twice the $\mathrm{IC}_{50}$ of selected quinolin-4(1H)-imines. We found that all compounds inhibit oxygen consumption in both promastigote and axenic amastigote forms, with higher efficiency in the latter (Fig. 1). Compound $1 \mathrm{n}$ was the most effective, decreasing oxygen consumption by around $65 \%$ in L. amazonensis promastigotes and by $85 \%$ in L. infantum axenic amastigotes. In contrast, compound $1 \mathrm{~h}$ showed milder effects on all species analyzed. In this case, oxygen consumption inhibition varied from $18 \%$ in L. amazonensis promastigotes to $39 \%$ in L. infantum axenic amastigotes. These results are consistent with the hypothesis that quinolin-4(1H)-imines may affect Leishmania respiratory complexes.

Quinolin-4(1H)-imines alter the mitochondrial membrane potential. Impairment of the electron transfer chain following inhibition of respiratory complexes (such as complex III) is often associated with the collapse of the mitochondrial membrane potential $\left(\Delta \Psi_{m}\right)$ (9). We used TMRE to investigate changes in $\Delta \Psi_{m}$ upon treatment of parasites with selected quinolin-4(1H)-imines. When incubated with cells, TMRE accumulates inside the negative environment of mitochondria, but after $\Delta \Psi_{\mathrm{m}}$ collapse, the probe 
A L. infantum axenic amastigotes

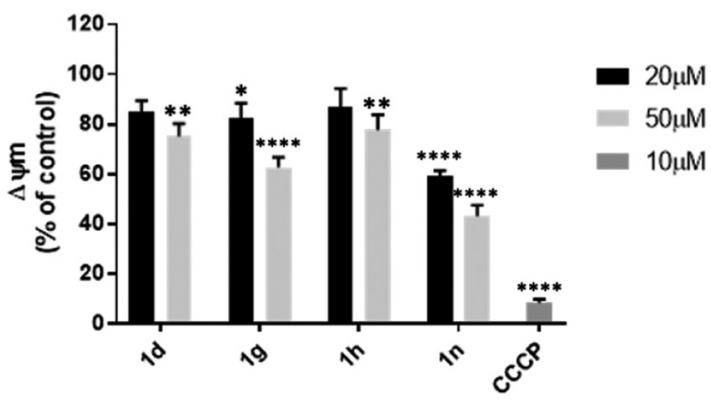

C L. amazonensis promastigotes

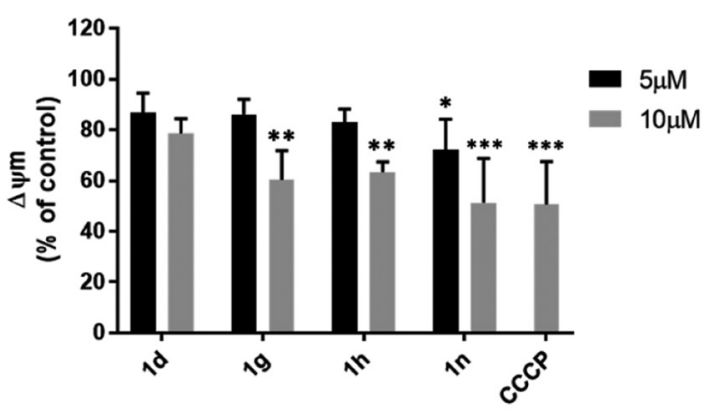

B

\section{L. infantum promastigotes}

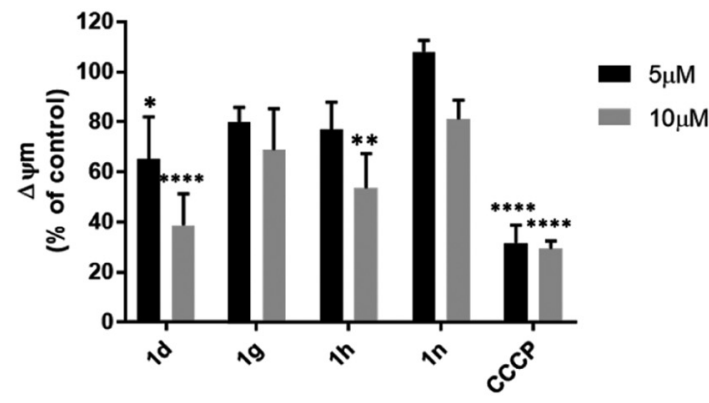

D L. major promastigotes

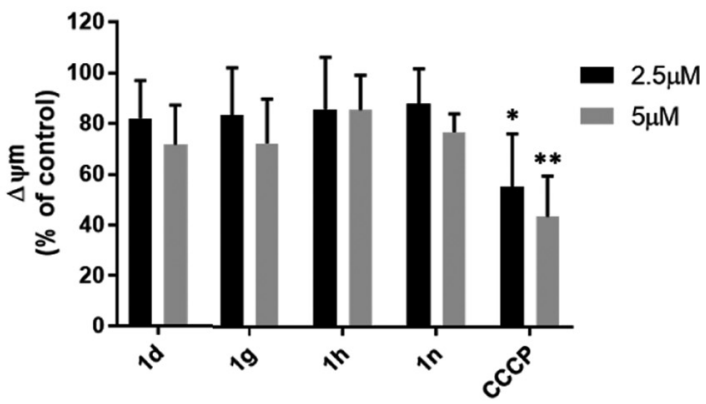

FIG 2 Effect of selected quinolin- $4(1 H)$-imines on mitochondrial membrane potential $(\Delta \Psi \mathrm{m})$ of Leishmania parasites. L. infantum axenic amastigotes (A) and L. infantum (B), L. amazonensis (C), and L. major (D) promastigotes were treated for 45 min with different concentrations of selected quinolin- $4(1 H)$-imines. CCCP, carbonyl cyanide $m$-chlorophenyl hydrazine, uncoupler of the $\Delta \Psi \mathrm{m}$, was used as the positive control. Graphs represent the mean fold change in treated parasites relative to control cells (treated with DMSO) and standard deviations (error bars) from 3 independent experiments. Statistical significance was determined using 2-way analysis of variance (ANOVA) with Sidak's multiple-comparison test. ${ }^{*}, P<0.05 ;{ }^{* *}, P<0.005 ;{ }^{* * *}, P<0.0005 ;{ }^{* * * *}, P<0.0001$.

diffuses out of the organelle, resulting in dissipation of the signal. We found that the $\Delta \Psi_{\mathrm{m}}$ of $L$. amazonensis (promastigotes) and $L$. infantum (promastigotes and axenic amastigotes) parasites was significantly decreased after incubation with quinolin-4(1H)-imines, similarly to incubation with CCCP, a potent uncoupler of the mitochondrial oxidative phosphorylation, used as a positive control (Fig. 2). In contrast, L. major (promastigotes) $\Delta \Psi_{\mathrm{m}}$ was not altered during the time frame of the analysis.

Quinolin-4(1H)-imines do not induce ROS production. We next evaluated ROS levels in parasites treated with selected quinolin- $4(1 H)$-imines, finding that none of the compounds triggered ROS generation (Fig. 3). This result is not inconsistent with activity of the compounds on one of the respiratory complexes, as the production of ROS depends on the exact site of the enzymatic complex that is targeted. In the case of complex III, for instance, inhibition of its $Q_{i}$ site (e.g., with antimycin $A$ ) generates reactive oxygen species, while interference with electron flow through the $Q_{0}$ site is not generally associated with the production of these species (15-17).

\section{DISCUSSION}

Current treatments for leishmaniases are based on chemotherapeutics that show important limitations in terms of efficacy, specificity, and administration regimens, rendering the development of new drugs a compelling necessity (4). Unfortunately, this faces significant challenges. First, Leishmania parasites in the disease-causing stage inhabit specific compartments within their mammalian host cells, which implies that, to reach the parasites, drugs must overcome two membrane barriers (18). Second, Leishmania parasites display an enormous capacity to adapt to the presence of exogenous compounds (4). Third, there is insufficient and unsustained investment by pharmaceutical companies in the development of drugs to treat leishmaniases, with much 
A

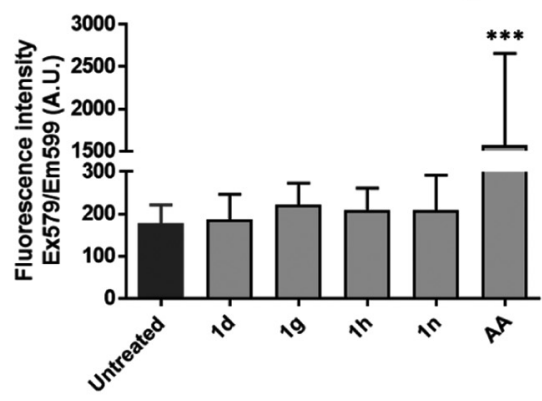

C

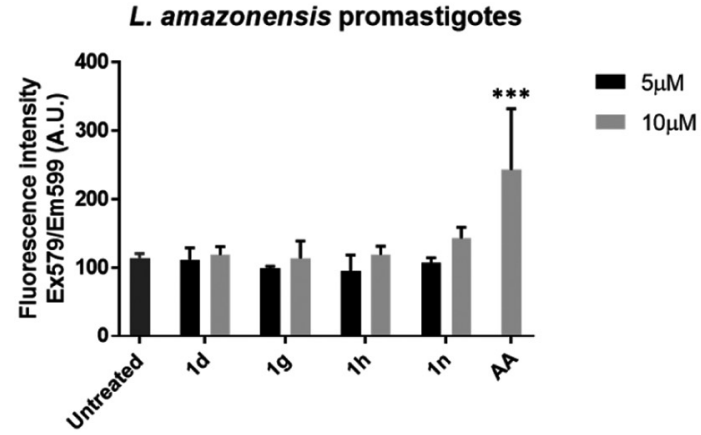

B

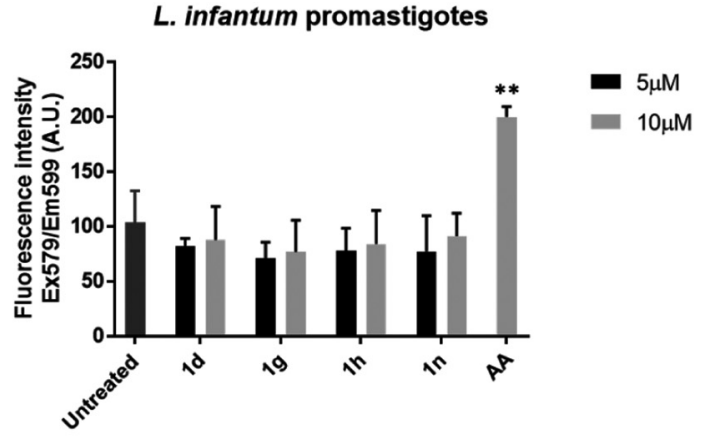

D

L. major promastigotes

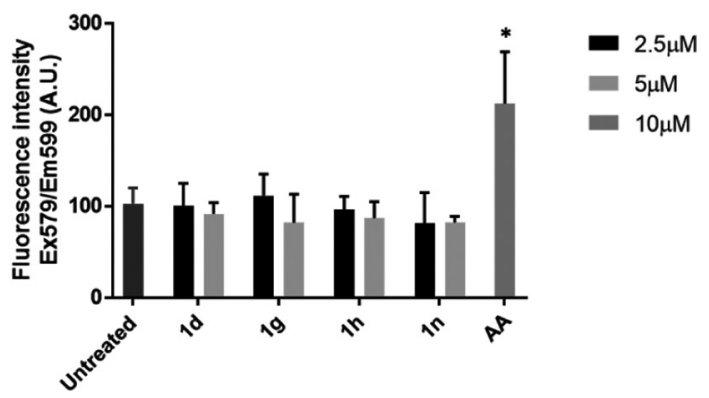

FIG 3 Analysis of ROS production in Leishmania parasites after the addition of selected quinolin-4(1H)-imines. $L$. infantum axenic amastigotes (A) and L. infantum (B), L. amazonensis (C), and L. major (D) promastigotes were treated with different concentrations of the selected quinolin-4(1H)-imines for $45 \mathrm{~min}$. AA, antimycin A, was used as the positive control for ROS production. Graphs show the mean values and standard deviations (error bars) from 3 independent experiments. Statistical significance was determined using 2way ANOVA with Sidak's multiple-comparison test. ${ }^{*}, P<0.05 ;{ }^{* *}, P<0.005 ;{ }^{* *}, P<0.0005 ;{ }^{* * *}, P<0.0001$.

of the effort toward this objective remaining academia driven (19). The present work, which represents an example of the latter studies, is aimed at assessing the antileishmanial value of a series of quinolin- $4(1 H)$-imine derivatives previously developed for inhibition of malaria parasites $(8,9)$.

The screening performed indicated that the quinolin- $4(1 H)$-imine scaffold is an interesting and novel chemotype for leishmaniasis drug development. Several of the compounds tested showed $\mathrm{IC}_{50} \mathrm{~s}$ toward intracellular $L$. infantum parasites in the low micromolar range, with two of them exhibiting $\mathrm{IC}_{50} \mathrm{~s}$ in the submicromolar range (compound $1 \mathrm{~h}, \mathrm{IC} \mathrm{C}_{50}=0.9 \mu \mathrm{M}$, and compound $1 \mathrm{n}, \mathrm{IC} \mathrm{C}_{50}=0.7 \mu \mathrm{M}$ ) and selectivity indexes of 11.1 and 9.7, respectively. Structure-activity relationships revealed that enhanced activity and selectivity are obtained with an ethyl group linked at the $\mathrm{N}-1$ nitrogen of the quinolonimine scaffold and that basic alkylamino side chains at this position are detrimental for activity (Table 1). Regarding the substituents at the imine moiety, groups with alkyl linkers between the two phenyl rings are, in general, beneficial for activity, although oxygen linkers are also tolerated. Inspection of the activity data against intramacrophagic amastigotes presented in Table 2 also reveals that compounds containing a chloro as an R1 group are more potent than their trifluoromethyl derivatives (e.g., compound $1 \mathrm{k}$ versus $1 \mathrm{e}$ and $1 \mathrm{n}$ versus $1 \mathrm{f}$ ). These findings suggest that the electronic nature of the group at the 7 position of the quinolonimine scaffold can be further explored in order to generate analogues with improved selectivity and physicochemical properties. Therefore, the future generation of quinolin- $4(1 H)$-imine libraries will explore the introduction of electron-donating groups and water-solubilizing groups at this position. Future work will also test the possibility of using specific systems to deliver those compounds in experimental settings.

While screening the different $L$. infantum forms, we observed that intramacrophagic amastigotes were more susceptible than axenic amastigotes to the quinolin-4(1H)-imines 
tested. Several explanations can account for this finding: (i) the compounds might display an inherent capacity to directly activate macrophages, (ii) drug metabolism within macrophages might induce the formation of a derivative more potent against intramacrophagic Leishmania than against axenic amastigotes, and (iii) there might be metabolic differences between the two parasite forms that render intramacrophagic amastigotes particularly prone to inhibition. Although we did not identify the mechanism responsible for the higher drug sensitivity of intracellular L. infantum parasites, our data, showing important differences in activity between different parasite forms, support studies advising for drug testing to be preferentially carried out on intramacrophagic amastigotes, a more physiological form of the parasite $(20,21)$.

Originally, the quinolin-4(1H)-imines tested were designed to favor hydrophobic interactions at the $Q_{0}$ binding pocket of the Plasmodium cytochrome $b c_{1}$ complex (9). However, it has been reported that the structurally related $4(1 H)$-pyridone $(22)$ and quinolone (23) antimalarials bind to the $Q_{i}$ site, suggesting that this binding site can accommodate a broader range of chemotypes than initially anticipated. As a means to approach the physiological target of the compounds under study in Leishmania parasites, we investigated their effect on parasite mitochondrial function. The robust inhibition of oxygen consumption observed suggests that in Leishmania parasites, the mechanism of action also involves inhibition of the mitochondrial electron transport chain. This is consistent with the alterations in $\Delta \Psi_{\mathrm{m}}$ observed in L. infantum and L. amazonensis parasites (albeit these were not detected in L. major parasites). Future investigations are required to confirm whether respiratory complexes, and specifically the complex III $\mathrm{Q}_{\mathrm{o}}$ site, are the primary targets of quinolin- $4(1 \mathrm{H})$-imines in Leishmania parasites. In this respect, it is interesting to notice that the compounds did not trigger ROS production, which is in line with what would be expected for a $Q_{0}$ inhibitor. Indeed, it is well established that, while drugs targeting the complex III $Q_{i}$ site (e.g., antimycin $A$ ) enhance superoxide production (15-17), $Q_{0}$ inhibitors (stigmatellin and myxothiazol) abolish or reduce ROS generation (15).

In conclusion, we have disclosed a new chemotype that expands the current toolbox of antileishmanial compounds with activity against intramacrophagic amastigotes. Despite the structural similarity between quinolin- $(1 H)$-imines and their quinoline counterparts, our previous research revealed that this particular chemotype displays low metabolic susceptibility. This contrasts with 8-aminoquinolines, such as primaquine and sitamaquine, which are metabolized by cytochrome P450 2D6 $(24,25)$ and generate hydroxyquinoline metabolites that have been associated with toxicity issues and glucose-6-phosphate dehydrogenase (G6PD) liability. Importantly, quinolin-4(1H)imines exert their effect, at least partially, by inhibiting mitochondrial respiration, a mechanism that remains underexplored in the context of antileishmanial therapy.

\section{MATERIALS AND METHODS}

Cells and parasites. Leishmania infantum promastigotes (MHOM/MA/67/ITMAP-263) were cultured at $25^{\circ} \mathrm{C}$ in RPMI 1640 GlutaMax supplemented with $10 \%$ (vol/vol) heat-inactivated fetal bovine serum (iFBS), $50 \mathrm{U} \cdot \mathrm{ml}^{-1}$ penicillin, $50 \mu \mathrm{g} \cdot \mathrm{ml}^{-1}$ streptomycin (from Gibco), and $20 \mathrm{mM} \mathrm{HEPES}$ sodium salt, $\mathrm{pH}$ 7.4 (Sigma). Leishmania major and Leishmania amazonensis promastigotes (MHOM/SA/85/JISH 118 and MHOM/BR/LTB0016, respectively) were maintained at $25^{\circ} \mathrm{C}$ in Schneider's insect medium (Sigma) supplemented with $10 \%$ (vol/vol) iFBS, $100 \mathrm{U} \cdot \mathrm{ml}^{-1}$ penicillin, $100 \mu \mathrm{g} \cdot \mathrm{ml}^{-1}$ streptomycin, $5 \mathrm{mM}$ HEPES sodium salt, $\mathrm{pH} 7.4$, and $5 \mu \mathrm{g} \cdot \mathrm{ml}^{-1}$ phenol red (Sigma). L. infantum axenic amastigotes were maintained at $37^{\circ}$ $\mathrm{C}$ and $5 \% \mathrm{CO}_{2}$ in MAA20 medium, as described previously (26). Briefly, MAA consisted of media 199 with Hanks' salts (Gibco) supplemented with $15 \mathrm{mM}$ D-glucose, $4 \mathrm{mM} \mathrm{NaHCO}{ }_{3}, 0.5 \%$ trypto-casein-soy (Biorad) and $25 \mathrm{mM}$ HEPES at a final $\mathrm{pH}$ of 6.5. Afterward, MAA20 was obtained by supplementing MAA medium with $20 \%$ (vol/vol) iFBS, $2 \mathrm{mM}$ GlutaMax (Gibco), and $0.023 \mathrm{mM}$ hemin.

Compound synthesis. The synthesis and characterization of all compounds screened has been previously reported $(9,10,13,14)$. Stock solutions with concentrations between 10 and $20 \mathrm{mM}$ were prepared by dissolving the compounds in dimethyl sulfoxide (DMSO) at room temperature and were stored at $-20^{\circ} \mathrm{C}$.

Evaluation of compound activity against promastigotes of different Leishmania species and axenic amastigotes of $L$. infantum. Promastigotes in the late exponential phase $\left(3 \times 10^{6} \mathrm{cell} / \mathrm{s} / \mathrm{ml}\right)$ were seeded in 96-well plates in a final volume of $100 \mu$ l of either RPMI (L. infantum) or Schneider's medium (L. major and L. amazonensis); L. infantum axenic amastigotes were seeded $\left(1.5 \times 10^{5}\right.$ cells $\left./ \mathrm{ml}\right)$ in $100 \mu \mathrm{l}$ of MAA20, also in 96-well plates. Stock solutions of test compounds were prepared in $100 \%$ DMSO. 
Parasites were then incubated with different concentrations (between 0.2 and $30 \mu \mathrm{M}$ ) of the compounds dissolved in medium (DMSO concentrations in wells never exceeded 0.5\%). DMSO (0.5\%) and amphotericin B were used as the negative and positive control, respectively. Parasite viability was evaluated upon $24 \mathrm{~h}$ of exposure to each compound and was assessed using resazurin (Sigma) to a final concentration of $0.1 \mathrm{mM}$ as described in Vale-Costa et al. (27). Fluorescence intensity (excitation at $530 \mathrm{~nm}$ and emission at $590 \mathrm{~nm}$ ) was measured in a Synergy Mx microplate reader (BioTek Instruments). The half-maximal inhibitory concentrations $\left(\mathrm{IC}_{50} \mathrm{~s}\right)$ were determined with GraphPad Prism 7 software (GraphPad Software, Inc., La Jolla, CA).

Generation of BMDM. Bone marrow-derived macrophages (BMDM) were obtained following a previously described protocol (28). Briefly, bone-marrow (BM) cells were isolated by flushing femurs and tibias of BALB/c mice with Dulbecco's modified Eagle's medium (DMEM; Gibco). Cells were collected, centrifuged, and suspended in DMEM supplemented with 10\% iFBS, $1 \%$ minimum essential mediumnonessential amino acids solution (MEM; Gibco), $50 \mathrm{U} \cdot \mathrm{ml}^{-1}$ penicillin, $50 \mu \mathrm{g} \cdot \mathrm{ml}^{-1}$ streptomycin (complete DMEM medium [CDMEM]), and 10\% L929 cell-conditioned medium (LCCM) as a source of macrophage-colony stimulating factor (M-CSF). BM cells were placed in petri dishes for $24 \mathrm{~h}$ at $37^{\circ} \mathrm{C}$ with $5 \%$ $\mathrm{CO}_{2}$. Nonadherent cells were then collected, counted, plated in 96-well plates ( 2.5 to $3 \times 10^{4}$ cells per well), and incubated at $37^{\circ} \mathrm{C}$ with $5 \% \mathrm{CO}_{2}$ for 10 days, with CDMEM plus $10 \% \mathrm{LCCM}$ renewal on the fourth and seventh days.

Determination of $\mathrm{IC}_{50} \mathrm{~s}$ against intramacrophagic $L$. infantum, L. major, and L. amazonensis. The activities of the compounds against intracellular amastigotes were determined in in vitro cultures of BMDM, in 96-well plates. Differentiated BMDM were infected with $L$. infantum axenic amastigotes or with $L$. major and L. amazonensis stationary-phase promastigotes at a multiplicity of infection (MOI) of 10. After $3 \mathrm{~h}$ of contact with macrophages, noninternalized parasites were removed by washing twice with DMEM and internalized parasites allowed to differentiate into amastigotes for $24 \mathrm{~h}$ prior to the addition of the compounds (at concentrations of 0.3 to $5 \mu \mathrm{M}$ ). Twenty-four hours later, cells were fixed with $4 \%$ paraformaldehyde, permeabilized with $0.1 \%$ Triton X-100, and stained with HCS CellMask deep red (Invitrogen) and DAPI (4',6-diamidino-2-phenylindole) (Sigma) for $30 \mathrm{~min}$ at room temperature. Cultures were imaged using the IN Cell Analyzer 2000 microscope (GE Healthcare) and analyzed with the IN Cell Investigator Developer Toolbox version 1.9.2 (GE Healthcare) for determination of the percentage of infection as previously described (28). Half-maximal inhibitory concentrations $\left(I C_{50} \mathrm{~s}\right)$ were calculated using GraphPad Prism 7 software.

Cytotoxicity on BMDM. The cytotoxicity of the compounds against mammalian cells was evaluated in BMDM isolated as described above. Macrophages were incubated with serial dilutions of the compounds (between 0.6 and $20 \mu \mathrm{M}$ ) for $24 \mathrm{~h}$ at $37^{\circ} \mathrm{C}$ with $5 \% \mathrm{CO}_{2}$. Cell viability was determined with the resazurin assay as described above. The percentages of viable BMDM were calculated in relation to control cultures to which only vehicle ( $0.5 \%$ DMSO) was added. Data were analyzed with GraphPad Prism 7 software, and the $50 \%$ cytotoxic concentration $\left(\mathrm{CC}_{50}\right)$ for each compound determined.

Determination of oxygen consumption in parasites. Oxygen consumption in stationary-phase promastigotes of L. infantum, L. major, and L. amazonensis, as well as in axenic amastigotes of L. infantum, was measured in a Clark-type oxygen electrode (Hansatech) at $25^{\circ} \mathrm{C}$ in respiration buffer $(300 \mathrm{mM}$ sucrose, $10 \mathrm{mM}$ potassium phosphate, $\mathrm{pH} 7.2,5 \mathrm{mM} \mathrm{MgCl}$, $10 \mathrm{mM} \mathrm{KCl}, 0.02 \%$ [wt/vol] bovine serum albumin [BSA], $1 \mathrm{mM}$ EGTA and $4 \mu \mathrm{M}$ carbonyl cyanide $m$-chlorophenyl hydrazine [CCCP]). For each reaction mixture, $100 \mu \mathrm{l}$ of a suspension of $3 \times 10^{8}$ parasites $/ \mathrm{ml}$ in PBS was used. Oxygen consumption was monitored continuously for $5 \mathrm{~min}$ as follows: basal oxygen consumption was read during $2 \mathrm{~min}$, compounds were added (at twice the $\mathrm{IC}_{50}$ ), and measurements were taken for 3 more minutes. One millimolar KCN, a complex IV inhibitor, was used as a control (complete inhibition of oxygen consumption). Results were evaluated with O2view software (Hansatech) (29).

Evaluation of mitochondrial membrane potential. Variation of the mitochondrial membrane potential $\left(\Delta \Psi_{\mathrm{m}}\right)$ in treated parasites was evaluated using the potential-sensitive probe tetramethylrhodamine ethyl ester (TMRE; Sigma). For this, $1 \times 10^{7}$ L. infantum, L. major, or L. amazonensis stationaryphase promastigotes or $L$. infantum axenic amastigotes were treated with the different compounds at concentrations of 2 to 5 times their $\mathrm{IC}_{50} \mathrm{~s}$ for $45 \mathrm{~min}$ and then incubated with $500 \mathrm{nM}$ TMRE for a further $30 \mathrm{~min}$. Treatments were carried out in the media (without iFBS) and temperature conditions described above. Upon two washes in medium (without iFBS), the $\Delta \Psi_{\mathrm{m}}$ was determined by monitoring fluorescence in a BioTek Synergy Mx microplate reader with excitation and emission wavelengths of $540 \mathrm{~nm}$ and $590 \mathrm{~nm}$, respectively. The mitochondrial oxidative phosphorylation uncoupler CCCP was used as a positive control.

Analysis of ROS production. Reactive oxygen species (ROS) production was determined using MitoTracker red $\mathrm{CM}-\mathrm{H}_{2} \mathrm{XRos}$ (Molecular Probes) according to the manufacturer's instructions. Briefly, $1 \times 10^{7}$ stationary-phase promastigotes of L. infantum, L. major, or L. amazonensis or $1 \times 10^{7}$ axenic amastigotes of $L$. infantum were treated with selected compounds (at concentrations of 2 to 5 times the $\mathrm{IC}_{50} \mathrm{~s}$ ) for $45 \mathrm{~min}$ and then incubated with $200 \mathrm{nM}$ MitoTracker red $\mathrm{CM}-\mathrm{H}_{2} \mathrm{XRos}$ for $30 \mathrm{~min}$. Subsequently, parasites were washed twice in their respective culture medium (without iFBS) and fluorescence quantified in a BioTek Synergy Mx microplate reader with excitation and emission wavelengths of $579 \mathrm{~nm}$ and $599 \mathrm{~nm}$, respectively. Antimycin A (AA) $(10 \mu \mathrm{M})$ was used as the positive control.

Ethical statement. The use of BALB/c mice was approved by the Local Animal Ethics Committee of i3S, licensed by DGAV (Direção Geral de Alimentação e Veterinária, Government of Portugal). Animals were handled in accordance with good animal practice as defined by national authorities (DGAV; Decree 113/2013 from 7th August) (30) and European legislation (Directive 2010/63/EU, revising Directive 86/ 609/EEC) (31). 


\section{ACKNOWLEDGMENTS}

This work was funded by the Portuguese Foundation for Science and Technology (FCT, Portugal) under the scope of the strategic funding of UIDB/04469/2020 unit, by the BioTecNorte operation (NORTE-01-0145-FEDER-000004) funded by the European Regional Development Fund under the scope of Norte 2020 Programa Operacional Regional do Norte, and by Fundação para a Ciência e Tecnologia through project grants number UID/DTP/ 04138/2019, PTDC/QEQ-MED/7097/2014, PTDC/MED-FAR/30266/2017, and PTDC/MED-QUI/ 30021/2017. It also received support from NORTE-01-0145-FEDER-000012 Structured Program on Bioengineered Therapies for Infectious Diseases and Tissue Regeneration, supported by Norte Portugal Regional Operational Program (NORTE 2020), under the PORTUGAL 2020 Partnership Agreement, through the European Regional Development Fund (ERDF). FCT provided additional support to H.C. and A.S.R. (Investigator FCT contracts number IF/01244/2015 and IF/01034/2014, respectively) and to A.G.G.A. (contract number $373 \mathrm{SFRH} / \mathrm{BD} / 93766 / 2013)$. We acknowledge the support of the i3S Scientific Platform BioSciences Screening, member of the national infrastructure PPBI-Portuguese Platform of Bioimaging (PPBI-POCI-01-0145-FEDER-022122) and PT-OPENSCREEN.

\section{REFERENCES}

1. World Health Organization. 2016. Leishmaniasis in high-burden countries: an epidemiological update based on data reported in 2014. Wkly Epidemiol Rec 91:287-296.

2. Uliana SRB, Trinconi CT, Coelho AC. 2018. Chemotherapy of leishmaniasis: present challenges. Parasitology 145:464-480. https://doi.org/10.1017/ S0031182016002523.

3. Monge-Maillo B, Lopez-Velez R. 2015. Miltefosine for visceral and cutaneous leishmaniasis: drug characteristics and evidence-based treatment recommendations. Clin Infect Dis 60:1398-1404. https://doi.org/10.1093/cid/ civ004.

4. Ponte-Sucre A, Gamarro F, Dujardin JC, Barrett MP, Lopez-Velez R, GarciaHernandez R, Pountain AW, Mwenechanya R, Papadopoulou B. 2017. Drug resistance and treatment failure in leishmaniasis: a 21 st century challenge. PLoS Negl Trop Dis 11:e0006052. https://doi.org/10.1371/ journal.pntd.0006052.

5. Burrows JN, Elliott RL, Kaneko T, Mowbray CE, Waterson D. 2014. The role of modern drug discovery in the fight against neglected and tropical diseases. MedChemCommun 5:688-700. https://doi.org/10.1039/c4md00011k.

6. Bongarzone S, Bolognesi ML. 2011. The concept of privileged structures in rational drug design: focus on acridine and quinoline scaffolds in neurodegenerative and protozoan diseases. Expert Opin Drug Discov 6:251-268. https://doi.org/10.1517/17460441.2011.550914.

7. Carvalho L, Luque-Ortega JR, Manzano Jl, Castanys S, Rivas L, Gamarro F. 2010. Tafenoquine, an antiplasmodial 8-aminoquinoline, targets leishmania respiratory complex III and induces apoptosis. Antimicrob Agents Chemother 54:5344-5351. https://doi.org/10.1128/AAC.00790-10.

8. Carvalho L, Luque-Ortega JR, Lopez-Martin C, Castanys S, Rivas L, Gamarro F. 2011. The 8-aminoquinoline analogue sitamaquine causes oxidative stress in Leishmania donovani promastigotes by targeting succinate dehydrogenase. Antimicrob Agents Chemother 55:4204-4210. https://doi.org/10.1128/AAC.00520-11.

9. Rodrigues T, da Cruz FP, Lafuente-Monasterio MJ, Goncalves D, Ressurreicao AS, Sitoe AR, Bronze MR, Gut J, Schneider G, Mota MM, Rosenthal PJ, Prudencio M, Gamo FJ, Lopes F, Moreira R. 2013. Quinolin-4(1H)-imines are potent antiplasmodial drugs targeting the liver stage of malaria. J Med Chem 56:4811-4815. https://doi.org/10.1021/jm400246e.

10. Ressurreicao AS, Goncalves D, Sitoe AR, Albuquerque IS, Gut J, Gois A, Goncalves LM, Bronze MR, Hanscheid T, Biagini GA, Rosenthal PJ, Prudencio M, O'Neill P, Mota MM, Lopes F, Moreira R. 2013. Structural optimization of quinolon-4(1H)-imines as dual-stage antimalarials: toward increased potency and metabolic stability. J Med Chem 56:7679-7690. https://doi.org/10.1021/ jm4011466.

11. Bachovchin KA, Sharma A, Bag S, Klug DM, Schneider KM, Singh B, Jalani HB, Buskes MJ, Mehta N, Tanghe S, Momper JD, Sciotti RJ, Rodriguez A, Mensa-Wilmot K, Pollastri MP, Ferrins L. 2019. Improvement of aqueous solubility of lapatinib-derived analogues: identification of a quinolinimine lead for human African Trypanosomiasis drug development. J Med Chem 62:665-687. https://doi.org/10.1021/acs.jmedchem.8b01365.
12. Ortiz D, Guiguemde WA, Hammill JT, Carrillo AK, Chen Y, Connelly M, Stalheim K, Elya C, Johnson A, Min J, Shelat A, Smithson DC, Yang L, Zhu F, Guy RK, Landfear SM. 2017. Discovery of novel, orally bioavailable, antileishmanial compounds using phenotypic screening. PLoS Negl Trop Dis 11:e0006157. https://doi.org/10.1371/journal.pntd.0006157.

13. Rodrigues T, Ressurreicao AS, da Cruz FP, Albuquerque IS, Gut J, Carrasco MP, Goncalves D, Guedes RC, dos Santos DJ, Mota MM, Rosenthal PJ, Moreira R, Prudencio M, Lopes F. 2013. Flavones as isosteres of $4(1 \mathrm{H})$ quinolones: discovery of ligand efficient and dual stage antimalarial lead compounds. Eur J Med Chem 69:872-880. https://doi.org/10.1016/j .ejmech.2013.09.008.

14. Rodrigues T, Guedes RC, dos Santos DJ, Carrasco M, Gut J, Rosenthal PJ, Moreira R, Lopes F. 2009. Design, synthesis and structure-activity relationships of (1H-pyridin-4-ylidene)amines as potential antimalarials. Bioorg Med Chem Lett 19:3476-3480. https://doi.org/10.1016/j.bmcl.2009.05 .017 .

15. Bleier L, Drose S. 2013. Superoxide generation by complex III: from mechanistic rationales to functional consequences. Biochim Biophys Acta 1827:1320-1331. https://doi.org/10.1016/j.bbabio.2012.12.002.

16. Quinlan CL, Gerencser AA, Treberg JR, Brand MD. 2011. The mechanism of superoxide production by the antimycin-inhibited mitochondrial Q-cycle. J Biol Chem 286:31361-31372. https://doi.org/10.1074/jbc.M111.267898.

17. Zorov DB, Juhaszova M, Sollott SJ. 2014. Mitochondrial reactive oxygen species (ROS) and ROS-induced ROS release. Physiol Rev 94:909-950. https://doi.org/10.1152/physrev.00026.2013.

18. Shahnaz G, Sarwar HS, Yasinzai M. 2018. Crossing biological barriers for leishmaniasis therapy: from nanomedicinal targeting perspective. In Afrin $\mathrm{F}$ (ed), Leishmaniases are re-emerging diseases, IntechOpen Limited, London, UK. https://doi.org/10.5772/intechopen.75911.

19. Weng HB, Chen HX, Wang MW. 2018. Innovation in neglected tropical disease drug discovery and development. Infect Dis Poverty 7:67. https://doi .org/10.1186/s40249-018-0444-1.

20. Vermeersch M, da Luz RI, Tote K, Timmermans JP, Cos P, Maes L. 2009. In vitro susceptibilities of Leishmania donovani promastigote and amastigote stages to antileishmanial reference drugs: practical relevance of stage-specific differences. Antimicrob Agents Chemother 53:3855-3859. https://doi.org/10.1128/AAC.00548-09.

21. De Rycker M, Hallyburton I, Thomas J, Campbell L, Wyllie S, Joshi D, Cameron S, Gilbert IH, Wyatt PG, Frearson JA, Fairlamb AH, Gray DW. 2013. Comparison of a high-throughput high-content intracellular Leishmania donovani assay with an axenic amastigote assay. Antimicrob Agents Chemother 57:2913-2922. https://doi.org/10.1128/AAC.02398-12.

22. Capper MJ, O'Neill PM, Fisher N, Strange RW, Moss D, Ward SA, Berry NG, Lawrenson AS, Hasnain SS, Biagini GA, Antonyuk SV. 2015. Antimalarial 4(1H)pyridones bind to the Qi site of cytochrome bc1. Proc Natl Acad Sci U S A 112:755-760. https://doi.org/10.1073/pnas.1416611112.

23. David Hong W, Leung SC, Amporndanai K, Davies J, Priestley RS, Nixon GL, Berry NG, Samar Hasnain S, Antonyuk S, Ward SA, Biagini GA, O'Neill 
PM. 2018. Potent antimalarial 2-pyrazolyl quinolone bc 1 (Qi) inhibitors with improved drug-like properties. ACS Med Chem Lett 9:1205-1210. https://doi.org/10.1021/acsmedchemlett.8b00371.

24. Camarda G, Jirawatcharadech $P$, Priestley RS, Saif A, March S, Wong MHL, Leung S, Miller AB, Baker DA, Alano P, Paine MJI, Bhatia SN, O'Neill PM Ward SA, Biagini GA. 2019. Antimalarial activity of primaquine operates via a two-step biochemical relay. Nat Commun 10:3226. https://doi.org/ 10.1038/s41467-019-11239-0.

25. Loiseau PM, Cojean S, Schrevel J. 2011. Sitamaquine as a putative antileishmanial drug candidate: from the mechanism of action to the risk of drug resistance. Parasite 18:115-119. https://doi.org/10.1051/parasite/ 2011182115.

26. Sereno D, Lemesre JL. 1997. Axenically cultured amastigote forms as an in vitro model for investigation of antileishmanial agents. Antimicrob Agents Chemother 41:972-976. https://doi.org/10.1128/AAC.41.5.972.

27. Vale-Costa S, Gomes-Pereira S, Teixeira CM, Rosa G, Rodrigues PN, Tomas A, Appelberg R, Gomes MS. 2013. Iron overload favors the elimination of Leishmania infantum from mouse tissues through interaction with reactive oxygen and nitrogen species. PLoS Negl Trop Dis 7:e2061. https://doi.org/10.1371/journal.pntd.0002061.

28. Gomes-Alves AG, Maia AF, Cruz T, Castro H, Tomas AM. 2018. Development of an automated image analysis protocol for quantification of intracellular forms of Leishmania spp. PLoS One 13:e0201747. https://doi.org/ 10.1371/journal.pone.0201747.

29. Melo AM, Duarte M, Moller IM, Prokisch H, Dolan PL, Pinto L, Nelson MA, Videira A. 2001. The external calcium-dependent NADPH dehydrogenase from Neurospora crassa mitochondria. J Biol Chem 276:3947-3951. https://doi.org/10.1074/jbc.M008199200.

30. Direção Geral de Alimentação e Veterinária, Ministério da Agricultura, do Mar, do Ambiente e do Ordenamento do Território. 2013. Decree-Law no. 113/2013 implementing EU Directive no. 2010/63 on animal protection for scientific purposes. Diário da República no. 151/2013, Série de 2013-87, p 4709-4739.

31. European Parliament and Council. 2010. Directive 2010/63/EU of the European Parliament and of the Council of 22 September 2010 on the protection of animals used for scientific purposes. Off J Eur Union L 276:33. 